\title{
Susceptibility-Weighted Imaging for Detection of Thrombus in Acute Cardioembolic Stroke
}

\author{
Min-Gyu Park, ${ }^{a}$ Se-Jin Oh, ${ }^{a}$ Seung Kug Baik, ${ }^{b}$ Dae Soo Jung, ${ }^{c}$ Kyung-Pil Park ${ }^{a}$ \\ ${ }^{a}$ Department of Neurology, Pusan National University Yangsan Hospital, Pusan National University School of Medicine, Research Institute for \\ Convergence of Biomedical Science and Technology, Yangsan, Korea \\ bepartment of Radiology, Pusan National University Yangsan Hospital, Pusan National University School of Medicine, Yangsan, Korea \\ 'Department of Neurology, Pusan National University Hospital, Pusan National University School of Medicine, Busan, Korea
}

Background and Purpose Susceptibility-weighted imaging (SWI) can show an intravascular thrombus as a hypointense susceptibility vessel sign (SVS). In this study, we investigated the usefulness of SWI in the detection of an intravascular thrombus in acute cardioembolic stroke by comparing the SVS on SWI to the vessel status on time-of-flight magnetic resonance angiography (MRA).

Methods We consecutively enrolled patients with cardioembolic stroke in the anterior circulation within 3 days from stroke onset. The frequency and location of the SVS on SWI were compared with those of occlusion on MRA.

Results One hundred and twenty-two patients were conclusively enrolled in this study. The SVS was observed in 75.4\% (92/122) of the enrolled patients. MRA showed occlusion in $57 \%$ (70/122) of the enrolled patients. The SVS was identified in all 70 patients with occlusion on MRA. The SVS was observed in $22(42.3 \%)$ of 52 patients without occlusion on MRA $(P<0.001)$, which was identified mainly in post-bifurcation segments of the middle cerebral artery: the M2 segment in 4 patients, M3 segment in 10 patients, M4 segment in 4 patients, A3 segment in 1 patient, and multiple segments in 2 patients. The mean length of the SVS in the M1 segment was $13.65 \mathrm{~mm}$ (median: $12.39 \mathrm{~mm}$, length range: 2.70-39.50 mm).

Conclusions SWI can provide useful information about the thrombus location, the presence of a single thrombus or multiple thrombi especially in distal intracranial arteries, and the thrombus burden, all in acute cardioembolic stroke.

Keywords Acute stroke; Diagnosis; Magnetic resonance imaging; Magnetic resonance angiography

\author{
Correspondence: Kyung-Pil Park \\ Department of Neurology, Pusan \\ National University Yangsan Hospital, \\ Pusan National University School of \\ Medicine, Research Institute for \\ Convergence of Biomedical Science and \\ Technology, 20 Geumo-ro, Mulgeum-eup, \\ Yangsan 50612, Korea \\ Tel: +82-55-360-2451 \\ Fax: +82-55-360-2152 \\ E-mail:kppark@pusan.ac.kr \\ Received: August 25, 2015 \\ Revised: November 4, 2015 \\ Accepted: November 5, 2015
}

The authors have no financial conflicts of interest.

\section{Introduction}

In acute cardioembolic stroke, successful recanalization is crucial because a failure of recanalization is closely associated with poor outcomes after thrombolysis. ${ }^{1}$ Recanalization by intravenous thrombolysis (IVT) can be influenced by the burden, composition, and location of a thrombus. ${ }^{2-7}$ It would be useful to identify the location and burden of a thrombus before
IVT in the planning of the strategy of recanalization therapy.

$\mathrm{T} 2$ *-weighted gradient echo imaging (GRE) and susceptibility-weighted imaging (SWI) have both been used to identify a thrombus in the form of hypointense signals within occluded arteries, which is termed a "susceptibility vessel sign" (SVS). ${ }^{6,8-10}$ A recent study has shown that SWI is more sensitive than GRE in the visualization of the SVS in the intracranial arteries during the acute phase of ischemic stroke. ${ }^{9}$ Further- 
more, SWI has been known to have similar sensitivity for the thrombus in proximal intracranial arteries and superior sensitivity for the thrombus in distal intracranial arteries to timeof-flight magnetic resonance angiography (MRA)..$^{10}$ These results can be attributed to the characteristics of the SWI sequence, such as the amplification of the $\mathrm{T} 2{ }^{*}$ signal drop by the deoxyhemoglobin of trapped red blood cells (RBCs) in the thrombus. ${ }^{11}$ A thrombus in cardioembolic stroke is known to be composed mainly of RBCs, and therefore, it can be well characterized by SWI.

For this reason, in the present study, we compared the SVS on SWI to the vessel status on MRA and analyzed the SVS frequency according to the time from stroke onset to the MR scan in acute cardioembolic stroke. We also assessed the thrombus burden by measuring the length of the SVS in proximal intracranial arteries.

\section{Methods}

\section{Patients}

We conducted a retrospective study on stroke patients admitted to our institute between March 2009 and March 2013. Patients were enrolled if they met the following inclusion criteria: (1) acute cerebral infarction in the anterior circulation confirmed by diffusion-weighted imaging, (2) cardioembolism determined by the Trial of Org 10172 in Acute Stroke Treatment classification, ${ }^{12}$ (3) MR imaging (MRI) performed within 3 days from stroke onset, and (4) MRI performed prior to thrombolytic therapy as long as the patients were treated with IVT or mechanical thrombectomy. We excluded patients with isolated intracranial internal carotid artery (ICA) occlusion, because it may be difficult to differentiate the SVS from susceptibility artifacts of the skull base. Patients with hemorrhagic transformation were excluded, because the SVS cannot be identified under hemorrhagic transformation. To identify the sources of cardioembolism, echocardiography (transthoracic or transesophageal) and 24-hour Holter monitoring were performed in enrolled patients who exhibited any of the following conditions: (1) a history of cardiac disease, (2) an irregular or abnormal rhythm on the electrocardiogram, (3) heart murmur, or (4) no relevant artery stenosis or atherosclerotic risk factors.

\section{MRI protocol}

All patients were examined using a $1.5 \mathrm{~T}$ or 3T MRI unit (Avanto, Verio, Skyra; Siemens, Erlangen, Germany). SWI was performed with the following relevant parameters at 1.5T: repetition time $(\mathrm{TR})=49 \mathrm{~ms}$, echo time $(\mathrm{TE})=40 \mathrm{~ms}$, flip an- gle $=15^{\circ}$, slice thickness $=2 \mathrm{~mm}$, intersection gap $=0 \mathrm{~mm}$, field of view $($ FOV $)=184 \times 210$, and matrix number $=320 \times 227$. Similarly, it was performed with the following relevant parameters at $3 \mathrm{~T}: \mathrm{TR}=28 \mathrm{~ms}, \mathrm{TE}=20 \mathrm{~ms}$, flip angle $=15^{\circ}$, slice thickness $=2 \mathrm{~mm}$, intersection gap $=0 \mathrm{~mm}, \mathrm{FOV}=184 \times 210$, and matrix number $=320 \times 227$. MRA was performed using a three-dimensional sequence with the following parameters at 3T: $\mathrm{TE}=3.9 \mathrm{~ms}, \mathrm{TR}=21 \mathrm{~ms}$, flip angle $=18^{\circ}, \mathrm{FOV}=168 \times 240$ $\mathrm{mm}$, slice thickness $=0.6 \mathrm{~mm}$, and pixel spacing $=0.53$; this resulted in an acquisition time of 2 minutes 59 seconds. Similarly, MRA was performed using a three-dimensional sequence with the following parameters at $1.5 \mathrm{~T}$ : $\mathrm{TE}=7 \mathrm{~ms}$, $\mathrm{TR}=24 \mathrm{~ms}$, flip angle $=25^{\circ}, \mathrm{FOV}=168 \times 280 \mathrm{~mm}$, slice thickness $=0.5 \mathrm{~mm}$, and pixel spacing $=0.625$; this resulted in an acquisition time 2 minutes 59 seconds.

\section{Imaging analysis}

The SWI were reviewed independently by two vascular neurologists (MG Park and KP Park) who were blinded to the clinical data and to other MR sequences. The SVS was defined as the hypointense signal on SWI within the vessels, exceeding the size of the homologous contralateral arterial diameter (Figure 1). The length of the SVS in the M1 segment was measured at the slice with the longest SVS. Vessel occlu-
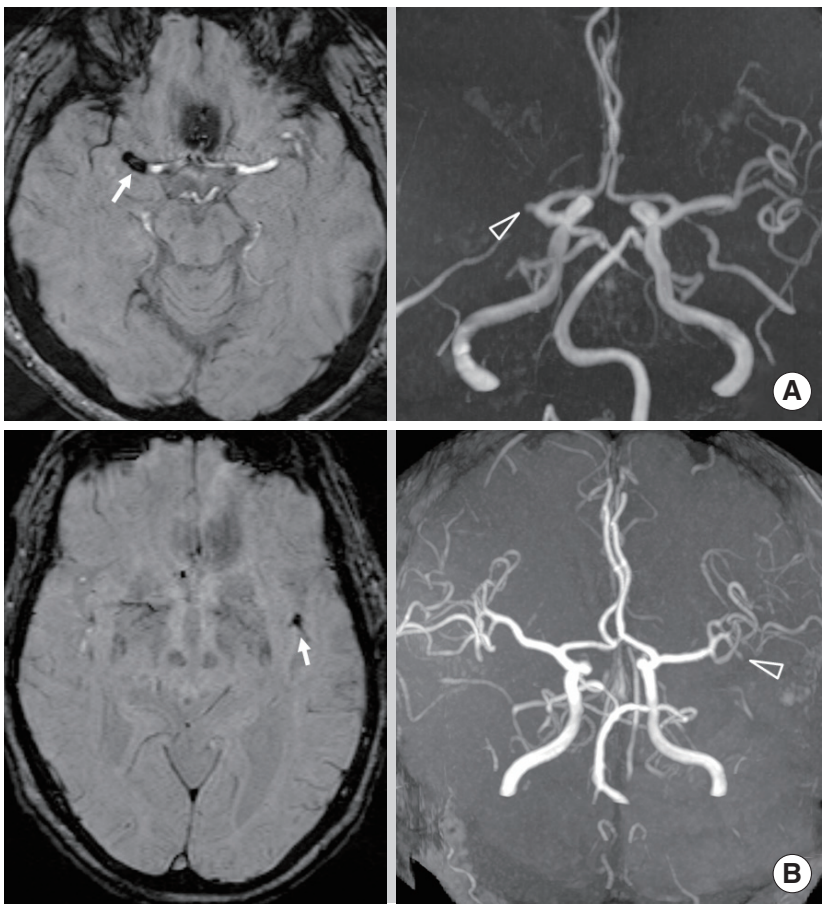

Figure 1. (A) Susceptibility-weighted imaging (SWI) shows susceptibility vessel sign (SVS, arrow) in M1 segment of right middle cerebral artery (MCA). MR angiography (MRA) shows occlusion (open arrowhead) in M1 segment of right MCA. (B) SWI shows SVS (arrow) in M2 segment of left MCA. MRA shows occlusion (open arrowhead) in left M2 segment. 
sion was defined as signal disruption on MRA (Figure 1). The MRA was independently reviewed by a vascular interventionist ( $\mathrm{SJ} \mathrm{Oh}$ ) who was blinded to the clinical data and to other MR sequences.

\section{Statistical analysis}

The McNemar test was performed with the aim of comparing the SVS on SWI with the occlusion on MRA. The interobserver variability of the SVS was analyzed by k-statistics. Pearson's $\chi^{2}$ test was performed for evaluating the frequency of the SVS in the time from stroke onset to the MR scan (i.e., $\leq 24$ hours and 24-72 hours). The Mann-Whitney U test was performed for comparing the frequencies of the SVS on SWI and the frequencies of the occlusions on MRA between the 1.5 $\mathrm{T}$ and 3.0 $\mathrm{T} \mathrm{MR}$ units. The data were analyzed using the Statistical Package for Social Sciences software for Windows (ver. 21; IBM, Armonk, New York). Statistical significance was defined as $P<0.05$.

\section{Results}

A total of 122 patients (including 71 females aged between 43 and 92 years) were finally enrolled in our study. The baseline characteristics of the patients are summarized in Table 1 . The

Table 1. Patient demographics

\begin{tabular}{lc}
\hline Characteristics & $\mathrm{N}=122$ \\
\hline Mean age (year) & $72.2(43-92)$ \\
Males & $51(41.8 \%)$ \\
Risk factors & \\
Hypertension & $71(58.2 \%)$ \\
Diabetes mellitus & $22(18.0 \%)$ \\
Hyperlipidemia & $21(17.2 \%)$ \\
Smoking & $23(18.9 \%)$ \\
History of angina/MI & $10(8.2 \%)$ \\
Congestive heart failure & $20(16.4 \%)$ \\
Previous TIA/stroke & $16(13.1 \%)$ \\
Initial NIHSS score & $12(0-25)$ \\
Causes of cardioembolism & \\
Paroxysmal AF & $17(13.9 \%)$ \\
Persistent AF & $89(73.0 \%)$ \\
Valvular AF & $13(10.7 \%)$ \\
Hypertrophic cardiomyopathy with AF & $3(2.4 \%)$ \\
Site of arterial occlusion on MRA (n = 70) & \\
M1 segment & $39(55.7 \%)$ \\
M2 segment & $14(20 \%)$ \\
M3 segment & $1(1.4 \%)$ \\
Distal ICA or carotid T-occlusion & $11(15.7 \%)$ \\
Multiple occlusion & $5(7.1 \%)$ \\
\hline
\end{tabular}

The numerals in parentheses are ranges or percentages.

$\mathrm{MI}$, myocardial infarction; TIA, transient ischemic attack; AF, atrial fibrillation; NIHSS, National Institutes of Health Stroke Scale; MRA, MR angiography; ICA, internal carotid artery. time interval from stroke onset to the MR scan ranged from 1.5 hours to 72 hours (mean: $19.3 \pm 13.6$ hours). MRI was performed on a 3.0T MR unit in 69.7\% (85/122) of the enrolled patients and on a $1.5 \mathrm{~T}$ MR unit in $30.3 \%(37 / 122)$ of the enrolled patients.

The SVS was detected in $75.4 \%(92 / 122)$ of the enrolled patients. Interpretation of the SVS showed good agreement between the neurologists $(\mathrm{k}=0.824, P<0.001)$. No significant difference in the frequency of the SVS was observed between the 1.5T MR unit and the 3.0T MR unit $(P=0.487): 75.7 \%$ $(28 / 37)$ in the $1.5 \mathrm{~T}$ MR unit and $75.3 \%(64 / 85)$ in the $3.0 \mathrm{~T}$ MR unit. Multiple SVSs were detected in 9 of 92 patients with the SVS (9.8\%). In 7 patients with multiple SVSs, all the SVSs were identified in the vascular territory distal to the primary occluding segment. In the remaining 2 patients with multiple SVSs, the SVSs were found in different vascular territories. MRA showed occlusions in 70 of 122 patients (57.4\%): occlusion of the M1 segment in 39 patients, of the M2 segment in 14 patients, of the $M 3$ segment in 1 patient, of the distal ICA in 5 patients, as well as carotid-T occlusion in 6 patients and multiple occlusions in 5 patients. No significant difference in the frequency of occlusion on MRA was observed between the 1.5T MR unit and the 3.0T MR unit $(P=0.272): 64.9 \%$ $(24 / 37)$ in the $1.5 \mathrm{~T}$ MR unit and $54.1 \%(46 / 85)$ in the 3.0T MR unit. The SVS was identified in all 70 patients with symptomatic occlusion on MRA (Table 2). In 2 patients with the M1 occlusion on MRA, the SVS was detected in not only the M1 segment but also the $M 3$ segment of one patient and in the P3 and A3 segments of the other patient: occlusion of the distal arteries was not detected on MRA. The representative case is shown in Figure 2. The SVS was observed in 22 (42.3\%) of 52 patients without occlusion on MRA $(P<0.001)$, which was identified mainly in the post-bifurcation segments of the MCA: in the M2 segment in 4 patients, in the M3 segment in 10 patients, in the M4 segment in 4 patients, in the $\mathrm{A} 3$ segment in 1 patient, and in multiple segments in 2 patients. The SVS was not detected in $24.6 \%$ (30/122) of the enrolled patients, who showed no occlusion on MRA. MRI was performed on 91 patients $(74.6 \%)$ within 24 hours from stroke onset and on 31 (26.4\%) patients $24-72$ hours after stroke onset: the SVS

Table 2. Comparison of susceptibility-weighted imaging and time-of-flight MR angiography for detecting arterial occlusion

\begin{tabular}{lccc} 
& Occlusion on MRA & No occlusion on MRA & Total \\
\hline SVS & 70 & 22 & 92 \\
No SVS & 0 & 30 & 30 \\
Total & 70 & 52 & 122
\end{tabular}

$P<0.001$ by McNemar test.

SVS, susceptibility vessel sign; MRA, MR angiography. 

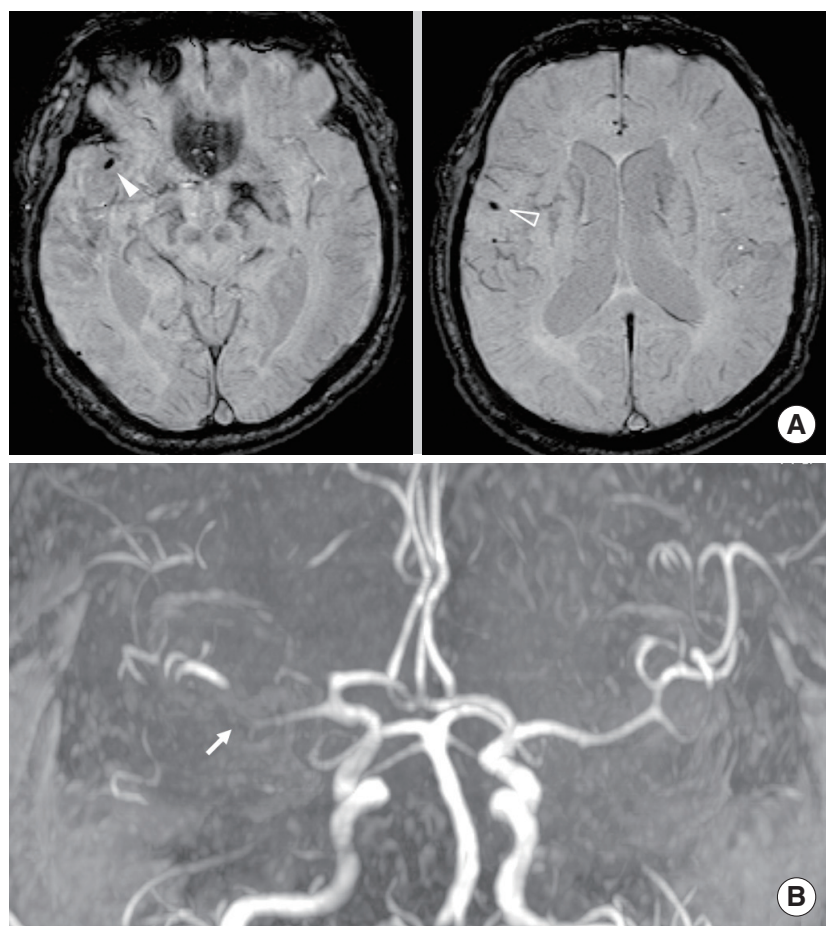

.

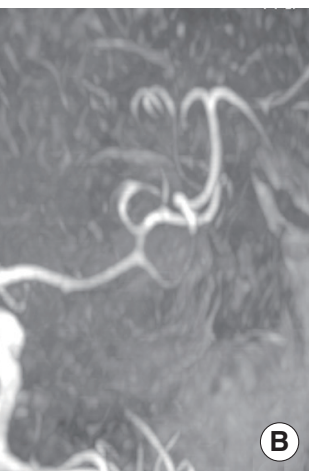

Figure 2. (A) Susceptibility-weighted imaging (SWI) shows multiple susceptibility vessel signs (SVSs) in distal M1 (arrowhead) and M3 (open arrowhead) segments of left middle cerebral artery (MCA). (B) MR angiography (MRA) does not show definite occlusion site except for distal M1 occlusion (arrow) of left MCA

was detected in $81.3 \%(74 / 91)$ and $20.2 \%(18 / 31)$ in each group (Figure 3, $P=0.009$ ).

The length of the SVS was measured in 49 patients with the thrombus in the M1 segment. The SVS length in the post-bifurcation segments was not measured, because these segments run vertically on the surface of the insula or obliquely on the cerebral cortex. The mean length of the SVS in the M1 segment was $13.65 \mathrm{~mm}$ (median: $12.39 \mathrm{~mm}$, length range: 2.70-39.50 mm).

\section{Discussion}

Our results show that SWI is more sensitive than MRA for detecting a thrombus in acute cardioembolic stroke, and it is especially sensitive for detecting a single thrombus or multiple thrombi in distal intracranial arteries. MRA is useful in evaluating intracranial arteries, but it has a limitation in terms of visualizing abnormalities of distal intracranial arteries because of its insensitivity to slow flow or slow in-plane flow. ${ }^{13}$ The SVS has been first reported through a study using T2*-weighted MRI. ${ }^{14}$ However, the detection rate of the SVS is inconsistent among previous studies conducted using GRE: the heterogeneous etiologies of the enrolled stroke patients may affect the inconsistent sensitivity of the SVS on GRE. ${ }^{13-15}$ The study of Cho et al.

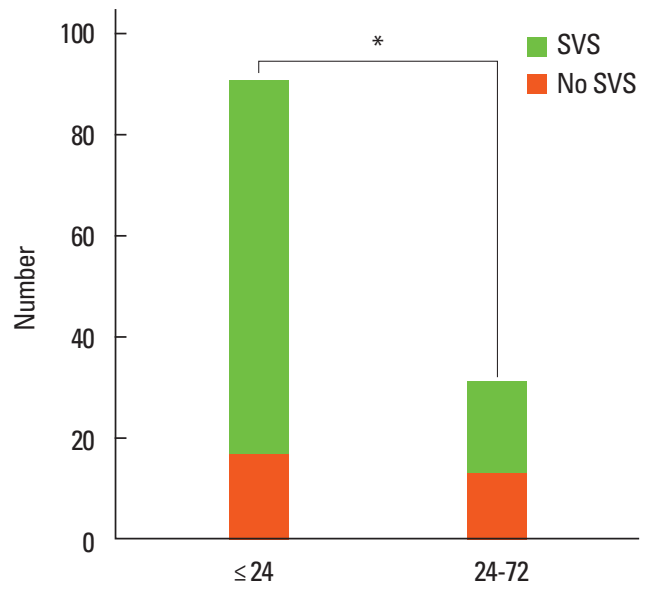

Time from stroke onset to MR scan (hour)

Figure 3. Frequency of susceptibility vessel sign (SVS) on susceptibilityweighted imaging in cardioembolic stroke patients in the time from stroke onset to MR scan. ${ }^{*} P=0.009$ by Pearson's chi-square test.

showed that the SVS on GRE was detected in $47.4 \%$ of 95 patients, especially in $77.5 \%$ of 40 patients with cardioembolic stroke. ${ }^{8}$ Furthermore, a recent study conducted using SWI in acute posterior cerebral artery infarction showed that cardioembolism is the most frequent etiology in patients with the SVS. ${ }^{17}$ Each human RBC contains approximately 270 million hemoglobin molecules. ${ }^{18}$ Fully deoxygenated hemoglobin has four unpaired electrons. If one $\mathrm{RBC}$ is completely unsaturated, one RBC will have approximately 1,080 million unpaired electrons, which are sources of a strong paramagnetic property. ${ }^{11}$ Therefore, in cardioembolic stroke, the higher detection rate of the SVS on SWI than that of occlusion on MRA is most probably because SWI can emphasize the "red thrombus" on account of the paramagnetic property induced by the unpaired electrons of deoxyhemoglobin.

In our study, 9.8\% of the enrolled patients showed multiple SVSs on SWI. MRA did not detect all of the multiple thrombi seen on the SVS, especially the distal thrombus. The limitation of MRA in the detection of multiple thrombi is attributable mainly to its dependence on blood flow, which fails to reach distal vessel occlusion on account of a decrease in or absence of blood flow through proximal vessel occlusion. A recent study assessed the prevalence of multiple thrombi before thrombolytic therapy in acute ischemic stroke by using SWI; multiple thrombi were detected on pretreatment SWI in 7.4\% of the patients with acute ischemic stroke, and the most frequent etiology in patients with multiple thrombi was cardioembolic stroke. ${ }^{19}$ Therefore, SWI is an appropriate sequence for evaluating the location and number of thrombi and for acute stroke imaging.

Our findings are similar to those of the study of Radbruch et 
al. in that they showed that SWI and MRA exhibit similar sensitivities for the detection of a thrombus in major arteries, whereas SWI is superior to MRA in the detection of a thrombus in distal arteries. However, because Radbruch et al. did not provide information about the stroke etiology of enrolled patients, it was unclear whether detection rate of SVS would be affected according to the stroke etiology..$^{10}$ The SVS frequency in our study was lower than that in their study (75.4\% in the former versus $92.6 \%$ in the latter). In their study, MR images were acquired within $4.6 \pm 2.5$ hours after the onset of symptoms in 33 patients with clear-onset stroke and within a maximum of 18 hours in 61 patients with unclear-onset stroke. ${ }^{10}$ Therefore, our lower detection rate of the SVS is most probably a result of later MR acquisition times than those in the study of Radbruch et al. Indeed, in our study, the SVS detection rate increased by $81.3 \%$ in patients within an early time window of 24 hours after stroke onset, which is higher than the SVS detection rate on GRE of cardioembolic stroke in the study of Cho et al. ${ }^{8}$ However, in our study, the SVS was not detected in $18.7 \%$ of the patients even though they were within an early time window; this can be explained by the occurrence of early spontaneous recanalization in cardioembolic stroke. It is well known that spontaneous recanalization frequently occurs in the early times of cardioembolic stroke. ${ }^{20}$ In fact, our study showed that the SVS frequency drops significantly 24 hours after stroke onset (Figure 2): early spontaneous recanalization may affect the negative detection of the SVS in patients within early time windows and the low detection rate of the SVS in patients within later time windows. Another possibility is that the thrombus was not detected on SWI, because it may be a white clot composed mainly of fibrin; ${ }^{21}$ however, even if the thrombus is a white clot, which cuts off the blood flow, MRA would be able to show vessel occlusion in at least the M1 segment. However, in our study, MRA could not show occlusion in all the patients without the SVS on SWI. Therefore, it is less likely that the thrombus was not detected on SWI because of the white clot.

Recently, Legrand et al. suggested that the clot burden score using the SVS on GRE is useful in the prediction of recanalization in hyperacute stroke patients treated by IVT. ${ }^{6}$ Previous studies have suggested that an $\mathrm{RBC}$-rich thrombus is more sensitive to IVT than is a platelet-rich thrombus. ${ }^{5,22}$ The number of RBCs in a thrombus is known to affect the susceptibility effect: the stronger the susceptibility effect, the higher is the number of RBCs that may be included in the thrombus. ${ }^{8,23}$ In our study, SWI was found to be more useful than MRA in defining the location of the thrombus and in determining its burden in the M1 segment. Therefore, although a recent study has
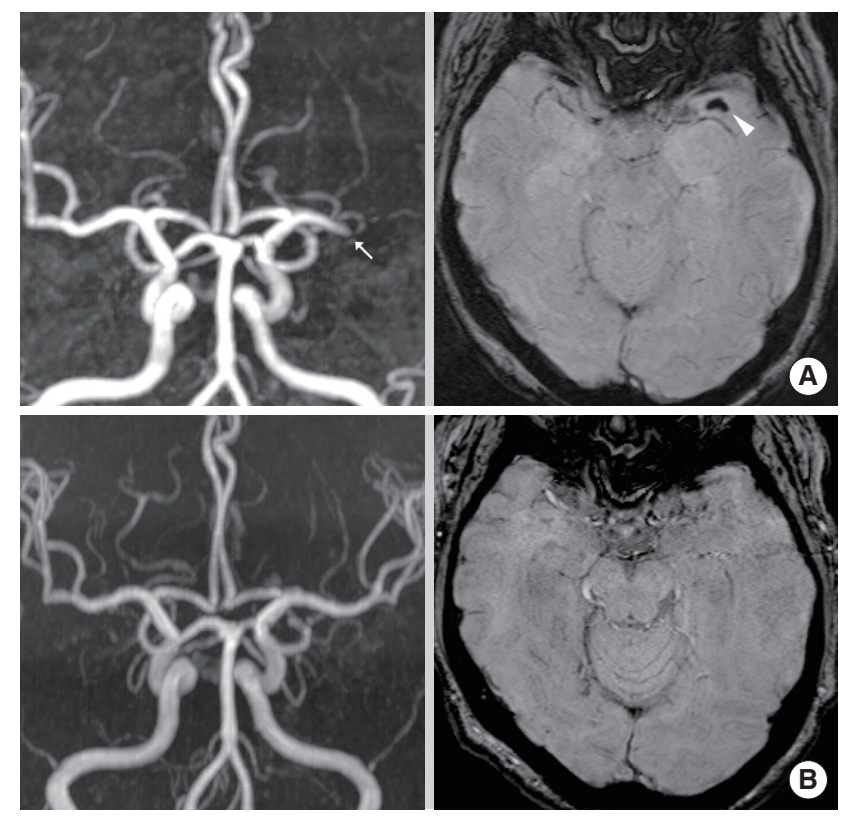

Figure 4. (A) 54 year-old woman (initial NIHSS score: 15) showed left M1 occlusion on MR angiography (MRA, arrow). Susceptibility-weighted imaging (SWI) before intravenous thrombolysis (IVT) shows susceptibility vessel sign (SVS, arrowhead) in left M1 segment. IVT was performed within $4 \mathrm{~h}$ from stroke onset. (B) Follow-up MRA after IVT shows recanalization of left M1. Follow-up SWI shows no SVS in left M1 segment. At 3 months, the modified Rankin scale of the patient was 0 .

shown that the thrombus burden on SWI does not affect the success of endovascular recanalization therapy, ${ }^{24}$ we suggest that further study is required to evaluate whether or not the response to IVT is affected by the thrombus burden using the SVS on SWI. ${ }^{6,7}$ A representative case is shown in Figure 4.

Our study had some limitations. First, we enrolled patients with cardioembolic stroke in later time windows. Therefore, further study will be required to confirm our results for cardioembolic stroke within the time window for thrombolytic therapy. Second, we enrolled patients of only cardioembolic stroke; therefore, our results cannot be generalized to stroke due to other etiologies. However, the characteristic of SVS on SWI considered in our study would be useful in understanding a thrombus due to cardioembolism. Third, 1.5T and 3T MR units were employed in our study. Although a higher field strength would enhance the detection of cerebral microbleeds on SWI, ${ }^{25}$ it is not known whether a higher field strength would affect the detection of the SVS: in our study, the SVS detection rate did not differ between the 1.5T and 3T MR units. Fourth, different field strengths of MR units may affect occlusion detection on MRA. ${ }^{26}$ 


\section{Conclusions}

SWI is highly useful in defining the location of a thrombus, especially of a single thrombus or of multiple thrombi in distal intracranial arteries, in acute cardioembolic stroke. In addition, SWI provides information about the thrombus burden and a distal intracranial thrombus, which cannot be obtained from MRA. We believe that SWI can be a useful MR sequence for assessing the thrombus location and burden in acute cardioembolic stroke.

\section{Acknowledgements}

This study was supported by the Research Institute for Convergence of biomedical science and technology Gran (30-2013-005), Pusan National University Yangsan Hospital.

We sincerely thank Chang O Son for the grammar check.

\section{References}

1. Nam HS, Lee KY, Kim YD, Choi HY, Cho HJ, Cha MJ, et al. Failure of complete recanalization is associated with poor outcome after cardioembolic stroke. Eur J Neurol 2011;18:11711178.

2. Beck C, Cheng B, Krützelmann A, Rosenkranz M, Gerloff C, Fiehler J, et al. Outcome of MRI-based intravenous thrombolysis in carotid-T occlusion. J Neurol 2012;259:2141-2146.

3. Aoki J, Kimura K, Shibazaki K, Sakamoto Y, Saji N, Uemura J. Location of the susceptibility vessel sign on T2*-weighted MRI and early recanalization within 1 hour after tissue plasminogen activator administration. Cerebrovasc Dis Extra 2013;3:111-120.

4. Linfante I, Llinas RH, Selim M, Chaves C, Kumar S, Parker $\mathrm{RA}$, et al. Clinical and vascular outcome in internal carotid artery versus middle cerebral artery occlusions after intravenous tissue plasminogen activator. Stroke 2012;33:2066-2071.

5. Jang IK, Gold HK, Ziskind AA, Fallon JT, Holt RE, Leinbach $\mathrm{RC}$, et al. Differential sensitivity of erythrocyte-rich and platelet-rich arterial thrombi to lysis with recombinant tissue-type plasminogen activator. A possible explanation for resistance to coronary thrombolysis. Circulation 1989;79:920-928.

6. Legrand L, Naggara O, Turc G, Mellerio C, Roca P, Calvet D, et al. Clot burden score on admission T2*-MRI predicts recanalization in acute stroke. Stroke 2013;44:1878-1884.

7. Riedel CH, Zimmermann P, Jensen-Kondering U, Stingele R, Deuschl G, Jansen O. The importance of size: successful recanalization by intravenous thrombolysis in acute anterior stroke depends on thrombus length. Stroke 2011;42:1775-1777.

8. Cho KH, Kim JS, Kwon SU, Cho AH, Kang DW. Significance of susceptibility vessel sign on $\mathrm{T} 2{ }^{*}$-weighted gradient echo imaging for identification of stroke subtypes. Stroke 2005;36: 2379-2383.

9. Allibert R, Billon Grand C, Vuillier F, Cattin F, Muzard E, Biondi A, et al. Advantages of susceptibility-weighted magnetic resonance sequences in the visualization of intravascular thrombi in acute ischemic stroke. Int J Stroke 2014;9:980-984.

10. Radbruch A, Mucke J, Schweser F, Deistung A, Ringleb PA, Ziener $\mathrm{CH}$, et al. Comparison of susceptibility weighted imaging and TOF-angiography for the detection of Thrombi in acute stroke. PloS One 2013;8:e63459.

11. Haacke EM, Mittal S, Wu Z, Neelavalli J, Cheng Y-CN. Susceptibility-weighted imaging: technical aspects and clinical applications, part 1. AJNR Am J Neuroradiol 2009;30:19-30.

12. Adams HP Jr, Bendixen BH, Kappelle LJ, Biller J, Love BB, Gordon DL, et al. Classification of subtype of acute ischemic stroke. Definitions for use in a multicenter clinical trial. TOAST. Trial of Org 10172 in Acute Stroke Treatment. Stroke 1993;24: 35-41.

13. Ishimaru H, Ochi M, Morikawa M, Takahata H, Matsuoka $Y$, Koshiishi T, et al. Accuracy of pre- and postcontrast 3D timeof-flight MR angiography in patients with acute ischemic stroke: correlation with catheter angiography. AJNR Am J Neuroradiol 2007;28:923-926.

14. Flacke S, Urbach H, Keller E, Träber F, Hartmann A, Textor J, et al. Middle cerebral artery (MCA) susceptibility sign at susceptibility-based perfusion MR imaging: clinical importance and comparison with hyperdense MCA sign at CT. Radiology $2000 ; 215: 476-482$.

15. Schellinger PD, Chalela JA, Kang D-W, Latour LL, Warach S. Diagnostic and prognostic value of early MR Imaging vessel signs in hyperacute stroke patients imaged $<3$ hours and treated with recombinant tissue plasminogen activator. AJNR Am J Neuroradiol 2005;26:618-624.

16. Assouline E, Benziane K, Reizine D, Guichard JP, Pico F, Merland JJ, et al. Intra-arterial thrombus visualized on $\mathrm{T} 2^{*}$ gradient echo imaging in acute ischemic stroke. Cerebrovasc Dis 2005; 20:6-11.

17. Park MG, Yoon CH, Baik SK, Park KP. Susceptibility vessel sign for intra-arterial thrombus in acute posterior cerebral artery infarction. J Stroke Cerebrovasc Dis 2015;24:1229-1234.

18. Kanias T, Acker JP. Biopreservation of red blood cells - the struggle with hemoglobin oxidation: biopreservation of RBC and hemoglobin oxidation. FEBS J 2010;277:343-356.

19. Gratz PP, Schroth G, Gralla J, Mattle HP, Fischer U, Jung S, et al. Whole-brain susceptibility-weighted thrombus imaging in stroke: fragmented thrombi predict worse outcome. AJNR Am J Neuroradiol 2015;36:1277-1282. 
20. Molina CA, Montaner J, Abilleira S, Ibarra B, Romero F, Arenillas JF, et al. Timing of spontaneous recanalization and risk of hemorrhagic transformation in acute cardioembolic stroke. Stroke 2001;32:1079-1084.

21. Liebeskind DS, Sanossian N, Yong WH, Starkman S, Tsang $\mathrm{MP}$, Moya AL, et al. CT and MRI early vessel signs reflect clot composition in acute stroke. Stroke 2011;42:1237-1243.

22. Niesten JM, van der Schaaf IC, van der Graaf Y, Kappelle LJ, Biessels GJ, Horsch AD, et al. Predictive value of thrombus attenuation on thin-slice non-contrast CT for persistent occlusion after intravenous thrombolysis. Cerebrovasc Dis 2014;37: 116-122.

23. Jha B, Kothari M. Pearls \& oy-sters: hyperdense or pseudohyperdense MCA sign: a Damocles sword? Neurology 2009;72: e116-e117.
24. Weisstanner C, Gratz PP, Schroth G, Verma RK, Köchl A, Jung $S$, et al. Thrombus imaging in acute stroke: correlation of thrombus length on susceptibility-weighted imaging with endovascular reperfusion success. Eur Radiol 2014;24:1735-1741.

25. Nandigam RN, Viswanathan A, Delgado P, Skehan ME, Smith $\mathrm{EE}$, Rosand J, et al. MR imaging detection of cerebral microbleeds: effect of susceptibility-weighted imaging, section thickness, and field strength. AJNR Am J Neuroradiol 2009;30:338343.

26. Choi CG, Lee DH, Lee JH, Pyun HW, Kang DW, Kwon SU, et al. Detection of intracranial atherosclerotic steno-occlusive disease with $3 \mathrm{D}$ time-of-flight magnetic resonance angiography with sensitivity encoding at 3T. AJNR Am J Neuroradiol 2007; 28:439-446. 